\title{
Numerical experiments for inverse analysis of material properties and size in functionally graded materials using the Artificial Bee Colony algorithm
}

\author{
H. Sakurai \\ Co-operative Education Center, \\ Sendai National College of Technology, Japan
}

\begin{abstract}
Functionally graded materials (FGMs) possess properties that vary gradually and are highly heat resistant. When incorporating these FGMs into a structure, the required distribution of material properties must be determined and produced to specification. Thus far, an inverse analysis method has been used for estimating a distribution of a Young's modulus of FGMs. However, minimizing an objective function to estimate a material property using the Davidon-FletcherPowell method did not converge under some initial conditions. Therefore, convergence of a solution depends on initial conditions. On the other hand, the Artificial Bee Colony (ABC) algorithm has drawn considerable interest in global optimization of a multimodal function. The objective of the present paper is to propose a method of numerical experimentation to conduct inverse analysis in FGMs with the ABC algorithm. A numerical experiment estimating both size and a graded index of an FGM beam that is based on measured stress values is presented. Next, determining a distribution of thermal conductivity in twodimensional FGMs using measured steady state temperatures is carried out. The results of the numerical experiments demonstrate the effectiveness of the proposed method.
\end{abstract}

Keywords: numerical experiment, inverse analysis, functionally graded material, graded index, thermal conductivity, Artificial Bee Colony algorithm. 


\section{Introduction}

Functionally graded materials (FGMs) possess properties that vary gradually and are highly heat resistant materials that can be used under high temperatures and high temperature gradient conditions. When incorporating these FGMs into a structure, the demands of the material properties must be determined and produced to specification. However, little research in this field has been published. Pioneering research, estimating the distribution of Young's modulus of FGMs using an inverse analysis method, has been published [1]. In addition, an inverse analytical technique using a finite element method and a numerical optimization technique to obtain material properties has been reported [2]. In the process of minimizing an objective function to estimate a material property using the Davidon-Fletcher-Powell method, the solutions did not converge under some initial conditions. Therefore, convergence of an optimizing solution depends on initial conditions [1]. Using such a gradient method system on a nonlinear optimization problem like FGM specification, might sometimes encounter a similar phenomenon.

On the other hand, in recent years, evolutionary algorithms have drawn considerable interest in global optimization of a multimodal function having many local minimum solutions. They are the Genetic Algorithm [3], the Immune Algorithm [4], Differential Evolution [5], the Ant Colony Optimization [6], and the Particle Swarm Optimization (PSO) [7]. Especially, it is reported that the Artificial Bee Colony (ABC) algorithm based on a particular intelligent behavior of honeybee swarms is a powerful method [8,9]. Furthermore, research on optimization of structures using evolutionary algorithms have also been published. Fourie and Groenwold [10] solved the minimum design problems of a plane truss, a space truss, and a torque arm by optimizing member sizes using the PSO method and also compared the calculation performance of GA and other gradient methods. Venter et al. [11] solved a problem of minimum volume of a cantilever beam subjected to a point load by PSO. Sonmez [12] used the ABC algorithm to solve the minimum weight design problems of plane and space trusses and showed the effectiveness of the method. Omkar et al. [13] studied multi-objective problems of composite materials based on the Vector Evaluated ABC algorithm. Hetmaniok et al. [14] carried out an inverse analysis of a boundary condition of heat flux in a one-dimensional heat conduction problem by the ABC algorithm using the distribution of unsteady state temperatures as input data. The purpose of this research is to ensure a specified strength under conditions of minimum weight and the total cost of components.

This paper proposes a method of numerical experimentation to carry out inverse analysis of functionally graded materials using the $\mathrm{ABC}$ algorithm. Two numerical experiments for inverse analysis in FGMs are conducted. First, a numerical experiment estimating both size and graded index in two-dimensional FGMs to satisfy measured multiple stress values is presented. Second, determining the distribution of thermal conductivity in two-dimensional FGMs based on multiple measured steady state temperatures is solved. Through these numerical experiments, the effectiveness of the proposed method is shown. 
Furthermore, when stresses or temperatures in inverse analysis are input as design conditions, the proposed method can be applied to optimization designs in FGMs.

\section{$2 \mathrm{ABC}$ algorithm and theoretical method of inverse analysis}

\subsection{ABC algorithm}

In this section, the $\mathrm{ABC}$ algorithm [8,9] will be described briefly. In the $\mathrm{ABC}$ algorithm, a colony of artificial bees consists of three groups of bees: employed bees, onlookers, and scout bees. Half of the colony is a group of employed bees, and the rest is a group of onlookers. The number of the employed bees is equal to the number of food sources. The employed bee of an abandoned food source becomes a scout bee. The $\mathrm{ABC}$ algorithm is comprised of the following searches:

(1) The employed bees locate a food source within the neighborhood of the hive. Employed bees share their information with onlookers within the hive.

(2) Based on information provided by the employed bees, the onlookers select a food source within the neighborhood of the food sources.

(3) An employed bee whose food source has been abandoned becomes a scout and starts to search for a new food source randomly.

\subsection{General theoretical method of inverse analysis}

This section describes the general theoretical method of this research. Consider the following objective function $f\left(c_{1}, c_{2}, \cdots, c_{n}\right)$ consisting of the residual sum of squares:

$$
f\left(c_{1}, c_{2}, \cdots, c_{n}\right)=\sum_{i=1}^{n_{m}}\left[u_{i}^{m}-u_{i}^{c}\left(c_{1}, c_{2}, \cdots, c_{n}\right)\right]^{2},
$$

where $u_{i}^{m}$ are physical quantities measured at the $n_{m}$ points and $u_{i}^{c}\left(c_{1}, c_{2}, \cdots, c_{n}\right)$ are physical quantities obtained by analysis that depend on the parameters to be determined. If the parameters $c_{1}, c_{2}, \cdots, c_{n}$ which minimize the objective function of eqn. (1) can be obtained, the inverse analysis can be performed. In minimizing the above objective function, the ABC algorithm is adopted. Furthermore, when the target design values are used instead of the measured values, it is possible to apply the present method to optimum design problems.

\subsection{Inverse analysis theory of graded index and size in two-dimensional FGMs}

In the Cartesian coordinate system, consider a cantilever FGM beam subjected to a transverse uniform load $p_{0}$ as shown in Figure 1. The length in the $x$ direction is $l$, and the height of the beam is $h$. This beam is assumed to be in the plane 
stress state. In Figure 1, the two question marks denote the unknowns of the inverse analysis.

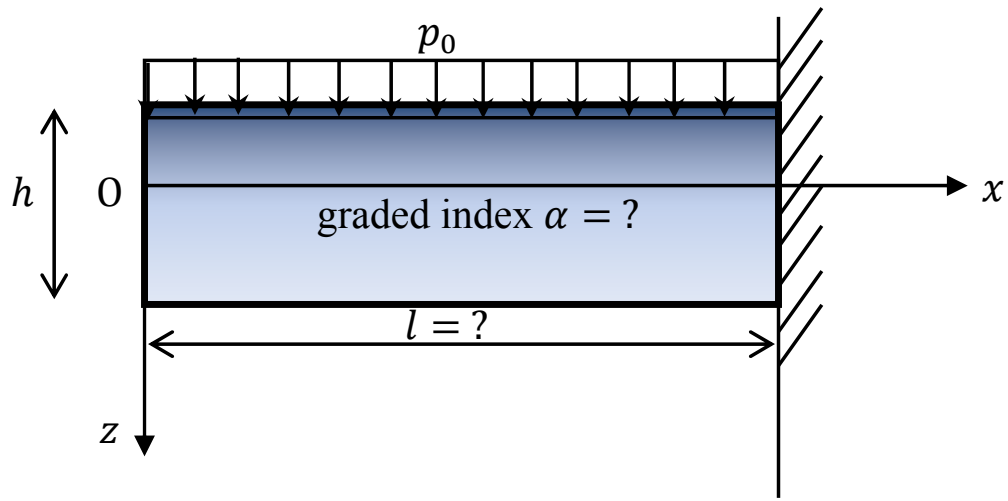

Figure 1: A cantilever FGM beam subjected to a uniform load.

The constitutive equations are given as:

$$
\begin{gathered}
\varepsilon_{x x}=S_{11} \sigma_{x x}+S_{13} \sigma_{z z} \\
\varepsilon_{z z}=S_{13} \sigma_{x x}+S_{33} \sigma_{z z} \\
\varepsilon_{z x}=S_{44} \sigma_{z x}
\end{gathered}
$$

where $\sigma_{x x}, \sigma_{z z}, \sigma_{z x}\left(\sigma_{z x}=\sigma_{x z}\right)$ are stress components, $\varepsilon_{x x}, \varepsilon_{z z}, \varepsilon_{z x}$ are strain components, and $S_{11}, S_{13}, S_{33}, S_{44}$ are elastic moduli. In this paper, we assume that the elastic moduli have same variations along $z$ direction, i.e.,

$$
S_{i j}=S_{i j}^{0} \cdot F(z)
$$

where $S_{i j}$ represents $S_{11}, S_{13}, S_{33}, S_{44}, S_{i j}^{0}$ are their corresponding values in the plane $z=z_{0}$ with $F\left(z_{0}\right)=1$ and $F(z)$ is called graded function. Further, we assume the graded function as:

$$
F(z)=e^{\frac{\alpha z}{h}}
$$

where the parameter $\alpha$ is the graded index [15]. Now, consider a problem in which both the graded index $\alpha$ and the length $l$ are unknown parameters and will be estimated from measured or prescribed stress values. In this case, the objective function in Eq. (1) is given as follows:

$$
f_{\sigma}(\alpha, l)=\sum_{i=1}^{n_{m}}\left[\sigma_{i}^{m}-\sigma_{i}^{c}(\alpha, l)\right]^{2},
$$

where $\sigma_{i}^{m}$ are stress values measured at the $n_{m}$ points, and $\sigma_{i}^{c}(\alpha, l)$ are the theoretical values calculated by stress analysis. 


\subsection{Inverse analysis theory of distribution of thermal conductivity in two-dimensional FGMs}

Next, consider a problem deducing the distribution of thermal conductivity in two-dimensional FGMs by the input distribution of steady state temperatures. In this problem, adopt the following eqn. (8) as the objective function to be minimized:

$$
f_{T}\left(a_{1}, a_{2}, \cdots, a_{n}\right)=\sum_{i=1}^{n_{m}}\left[T_{i}^{m}-T_{i}^{c}\left(a_{1}, a_{2}, \cdots, a_{n}\right)\right]^{2},
$$

where $T_{i}^{m}$ are steady state temperatures measured at the $n_{m}$ points, and $T_{i}^{c}\left(a_{1}, a_{2}, \cdots, a_{n}\right)$ are temperatures to be obtained by steady state heat conduction analysis. The temperatures $T_{i}^{c}\left(a_{1}, a_{2}, \cdots, a_{n}\right)$ depend on parameters $a_{1}, a_{2}, \cdots, a_{n}$ which express distribution of the thermal conductivity. Trying to estimate the values of the thermal conductivity at many points discretely in the domain of FGM leads to increasing the number of unknowns and CPU time. Instead, express the distribution of the thermal conductivity $\lambda(x, y)$ of FGMs by the following arbitrary base functions $\lambda_{i}(x, y)$ :

$$
\lambda(x, y)=a_{0}+a_{1} \lambda_{1}(x, y)+a_{2} \lambda_{2}(x, y)+\cdots+a_{n} \lambda_{n}(x, y) .
$$

In the above equation, as to be stated in 3.2 , such as power functions can be used as the functions $\lambda_{i}(x, y)$. Of course, it's necessary to choose appropriate functions depending on a problem to be solved. At this time, the unknowns will be the coefficients $a_{0}, a_{1} \cdots$. Another advantage of the method is its ability to obtain the distribution of the thermal conductivity continuously in addition to reducing unknowns and CPU time. In eqn. (8), the measured temperatures are assumed to be steady state temperatures, so an arbitrary value may be assigned to coefficient $a_{0}$ because the coefficient $a_{0}$ affects only the time to reach steady state.

\section{Numerical experiments and discussion}

\subsection{Numerical experiment on inverse analysis of a graded index and length of cantilever FGM beam}

A numerical experiment on the inverse analysis of the graded index and the length of the cantilever FGM beam shown in Figure 1 was conducted. The material properties at $z=z_{0}=0$ are given in Table 1 [15]. In the plane stress analysis, the analytical method [16] was used. In addition, the stress values obtained by the forward analysis were used as the measured stresses $\sigma_{i}^{m}$ in eqn. (7). The thickness of the beam $h$ and the magnitude of the transverse uniform pressure $p_{0}$ were set to $h=0.2[\mathrm{~m}], p_{0}=1[\mathrm{~Pa}]$. 
Table 1: $\quad$ Material properties $S_{i j}^{0}$ in eqns. (5) and (6).

\begin{tabular}{|c|c|}
\hline Material constants & Values $[1 / \mathrm{Pa}]$ \\
\hline$S_{11}^{0}$ & $5.41 \times 10^{-12}$ \\
\hline$S_{13}^{0}$ & $1.51 \times 10^{-12}$ \\
\hline$S_{33}^{0}$ & $9.52 \times 10^{-11}$ \\
\hline$S_{44}^{0}$ & $1.37 \times 10^{-10}$ \\
\hline
\end{tabular}

The normal stresses $\sigma_{x x}$ at eleven points on $x=l$ were used as measured stresses. Also, the graded index values were $\pm \alpha=3$. Figure 2 shows the distribution of stress $\sigma_{x x}$ in the $z$ direction used in the numerical experiment.

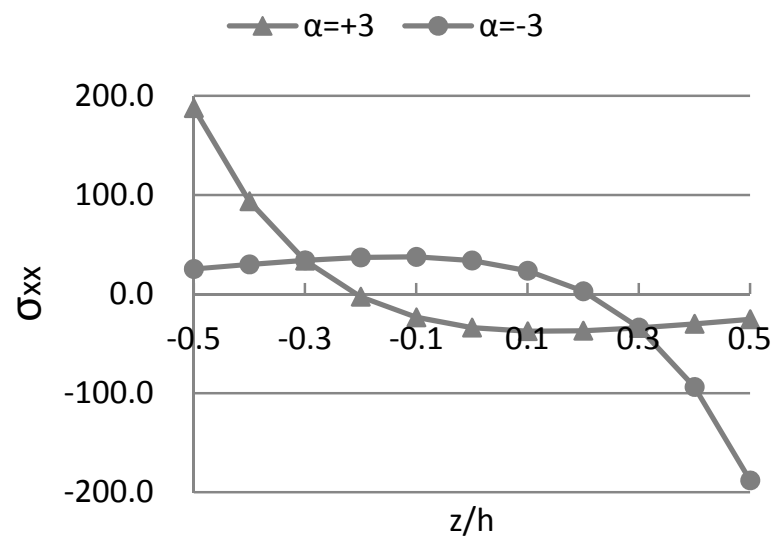

Figure 2: Measured stresses $\sigma_{x x}$ through the thickness of cantilever FGM beam at clamped end $x=l$

The control parameters in the ABC algorithm are shown in Table 2. The parameter $C_{\text {limit }}$ controls changing employed bees to scout bees.

Table 2: Control parameters used in the ABC algorithm.

\begin{tabular}{|c|c|}
\hline Colony size $(N)$ & 60 \\
\hline Number of employed bees $(S N)$ & 30 \\
\hline Number of onlookers $(N-S N)$ & 30 \\
\hline$C_{\text {limit }}$ & $0.1 \times S N \times D$ \\
\hline
\end{tabular}

In this numerical experiment, constraint conditions $-5 \leq \alpha \leq+5$ and $0.5 \leq l \leq 2.0$ were imposed on the graded index and the length of the beam, respectively. The best and the worst solutions and the corresponding objective function values $f_{\sigma}(\alpha, l)$ at each step are shown Table 3 . 
Table 3: Estimated $\alpha, l$ and objective function values $f_{\sigma}(\alpha, l)$.

(a) Case in which accurate values are given by $\alpha=+3.0, l=1.0$

\begin{tabular}{|c|c|c|c|}
\hline Step number & $\alpha, l$ and $f_{\sigma}(\alpha, l)$ & Best solution & Worst solution \\
\hline \multirow{3}{*}{100} & $\alpha$ & 2.94 & 2.56 \\
\cline { 2 - 4 } & $l$ & 1.01 & 0.92 \\
\cline { 2 - 4 } & $f_{\sigma}(\alpha, l)$ & $5.19 \times 10^{1}$ & $1.06 \times 10^{4}$ \\
\hline \multirow{3}{*}{150} & $\alpha$ & 2.96 & 2.94 \\
\cline { 2 - 4 } & $l$ & 1.01 & 0.99 \\
\cline { 2 - 4 } & $f_{\sigma}(\alpha, l)$ & $5.02 \times 10^{0}$ & $9.31 \times 10^{2}$ \\
\hline \multirow{3}{*}{300} & $\alpha$ & 2.99 & 2.96 \\
\cline { 2 - 4 } & $l$ & 1.00 & 1.00 \\
\cline { 2 - 4 } & $f_{\sigma}(\alpha, l)$ & $3.28 \times 10^{-2}$ & $5.78 \times 10^{0}$ \\
\hline
\end{tabular}

(b) Case in which accurate values are given by $\alpha=-3.0, l=1.0$

\begin{tabular}{|c|c|c|c|}
\hline Step number & $\alpha, l$ and $f_{\sigma}(\alpha, l)$ & Best solution & Worst solution \\
\hline \multirow{3}{*}{100} & $\alpha$ & -2.88 & -2.38 \\
\cline { 2 - 4 } & $l$ & 1.03 & 0.97 \\
\cline { 2 - 4 } & $f_{\sigma}(\alpha, l)$ & $1.95 \times 10^{2}$ & $2.26 \times 10^{4}$ \\
\hline \multirow{3}{*}{150} & $\alpha$ & -2.99 & -3.39 \\
\cline { 2 - 4 } & $l$ & 0.98 & 0.92 \\
\cline { 2 - 4 } & $f_{\sigma}(\alpha, l)$ & $3.13 \times 10^{1}$ & $9.85 \times 10^{2}$ \\
\hline \multirow{3}{*}{300} & $\alpha$ & -3.01 & -3.06 \\
\cline { 2 - 4 } & $l$ & 0.99 & 0.98 \\
\cline { 2 - 4 } & $f_{\sigma}(\alpha, l)$ & $2.16 \times 10^{0}$ & $1.93 \times 10^{1}$ \\
\hline
\end{tabular}

In both cases, the best solutions converged to the approximate solutions around step 150. Furthermore, all the employed bees converged to the approximate values with high accuracy at step 300. In this problem, stress was given as a measured quantity, however if it is not practical to give the stress as a measured quantity, another physical quantity like strain must be considered. This example was the inverse analysis of the cantilever FGM beam subjected to uniform pressure. However, this method may be applicable to inverse analysis of more general FGM structures.

\subsection{Numerical experiment on inverse analysis of distribution of thermal conductivity in two-dimensional FGM}

This section estimates the distribution of thermal conductivity of a twodimensional FGM based on a distribution of steady state temperatures. Figure 3 shows the heat conduction problem of the two-dimensional FGMs over a square domain $D=\{(x, y): 0 \leq x \leq 1,0 \leq y \leq 1\}$. The boundary conditions are set to $T=283[\mathrm{~K}]$ at $x=0, T=273[\mathrm{~K}]$ at $x=1$, and insulation conditions are imposed on both the upper and lower sides [17,18]. In the figure, the term $\partial T / \partial n$ denotes the differential of the normal direction. As later, points where 
temperatures were measured are located within the domain surrounded by the white dotted line. The color gradation in the domain expresses the variation of the thermal conductivity of the FGM. Additionally, " $\lambda=$ ?" means that the distribution of the thermal conductivity is unknown and its quantity was estimated by the numerical experiment.

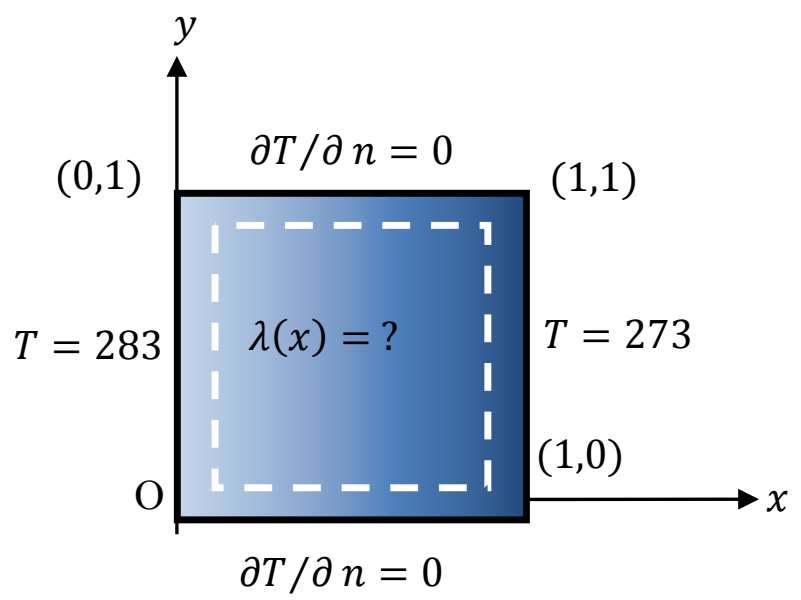

Figure 3: Geometry, boundary conditions, and measured domain of temperatures.

It is assumed that the true distribution of the thermal conductivity of the FGMs is expressed by the following equation:

$$
\lambda(x)=\exp (B x)
$$

where $B$ is a constant.

The goal is to obtain the approximate distribution of the thermal conductivity from the measured steady state temperatures. The distribution of the thermal conductivity of eqn. (10) depends upon only the $x$ coordinate, therefore, the following power function was used as the base function $\lambda_{i}(x, y)$ in eqn. (9).

$$
\lambda(x)=\sum_{i=0}^{3} a_{i} x^{i}=a_{0}+a_{1} x+a_{2} x^{2}+a_{3} x^{3}
$$

As stated in 2.4, an arbitrary value can be assigned to coefficient $a_{0}$. Therefore the value of $a_{0}$ was set to $a_{0}=1$. In a similar manner, the thermal capacity $\rho c$, the product of the thermal conductivity $\rho$, and the specific heat $\mathrm{c}$ can also take arbitrary constants so $\rho c=1$ in the steady state heat conduction analysis.

The temperatures of the forward analysis were used for the measured temperature distribution. The forward analysis was performed on $\mathrm{B}=+2$ in eqn. (10). Twenty-five measured points were placed equally in the $x$ direction 
and the $y$ direction within the domain $D=\{(x, y): 0.1 \leq x \leq 0.9,0.1 \leq y \leq$ $0.9\}$. Figure 4 shows the distribution of the measured temperatures along $y=0.5$.

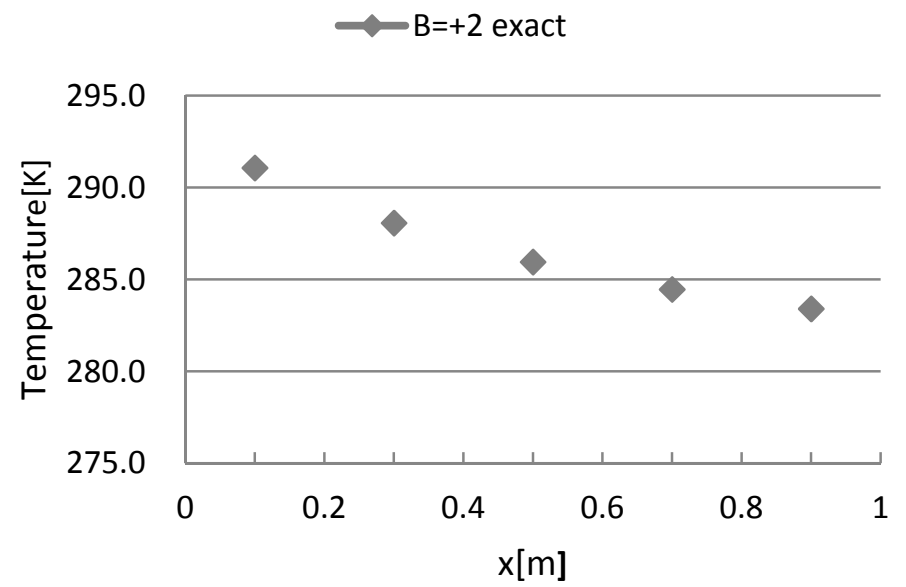

Figure 4: Measured distribution of steady-state temperature along $y=0.5$.

In this numerical experiment, the constraint conditions imposed were $0 \leq a_{1}, a_{2}, a_{3} \leq 5$ with respect to the coefficients. The discretization in the Moving Particle Semi-Implicit method used a total of 121 particles located uniformly in the domain in the steady state heat conduction analysis [18-20]. The control parameters of the $\mathrm{ABC}$ algorithm used in the present experiment are shown in Table 4.

Table 4: Control parameters used in the ABC algorithm.

\begin{tabular}{|c|c|}
\hline Colony size $(N)$ & 40 \\
\hline Number of employed bees $(S N)$ & 20 \\
\hline Number of onlookers $(N-S N)$ & 20 \\
\hline$C_{\text {limit }}$ & $0.1 \times S N \times D$ \\
\hline
\end{tabular}

The distribution of the thermal conductivity estimated by the numerical experiment is shown in Figure 5, where "accurate distribution" is the distribution of the thermal conductivity computed by eqn. (10), and "estimated distribution" is obtained by the present numerical experiment. Figure 6 shows distributions of the employed bees at the initial step and the $100^{\text {th }}$ step in $a_{1} a_{2}$ space.

The maximum relative error of the 11 points plotted in Figure 5 is within $4 \%$. Hence, the solution can be considered to have good accuracy compared to the correct solution. This study dealt with the steady state problem; however 
the resent numerical experiment method may be also applicable to an unsteady state problem.

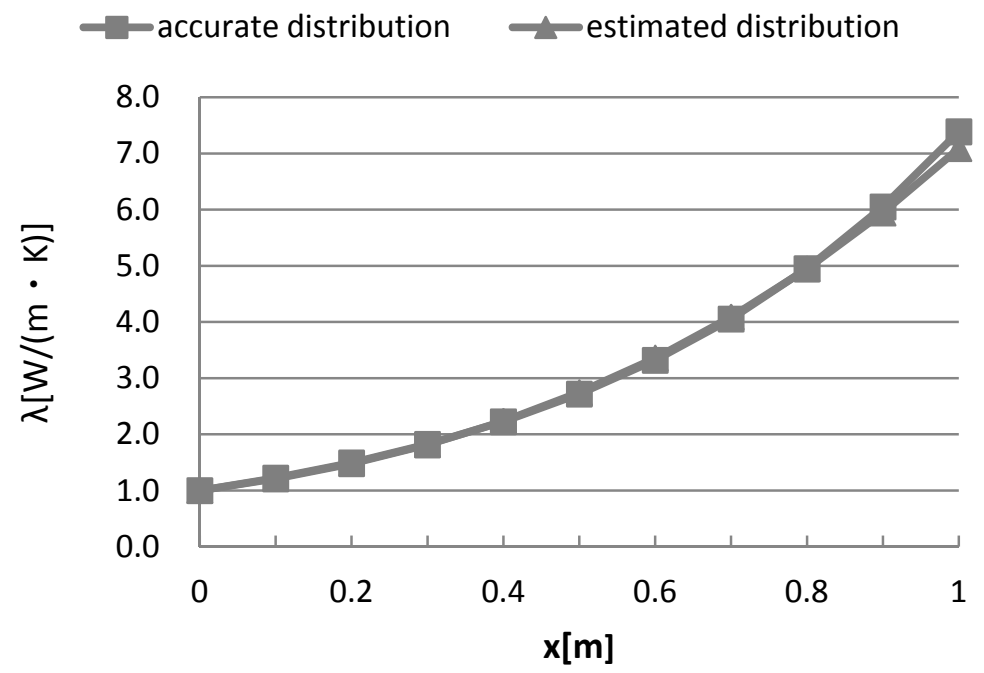

Figure 5: Estimated distribution of thermal conductivity of the two-dimensional FGM.

$\checkmark$ initial step $\quad$ 100th step

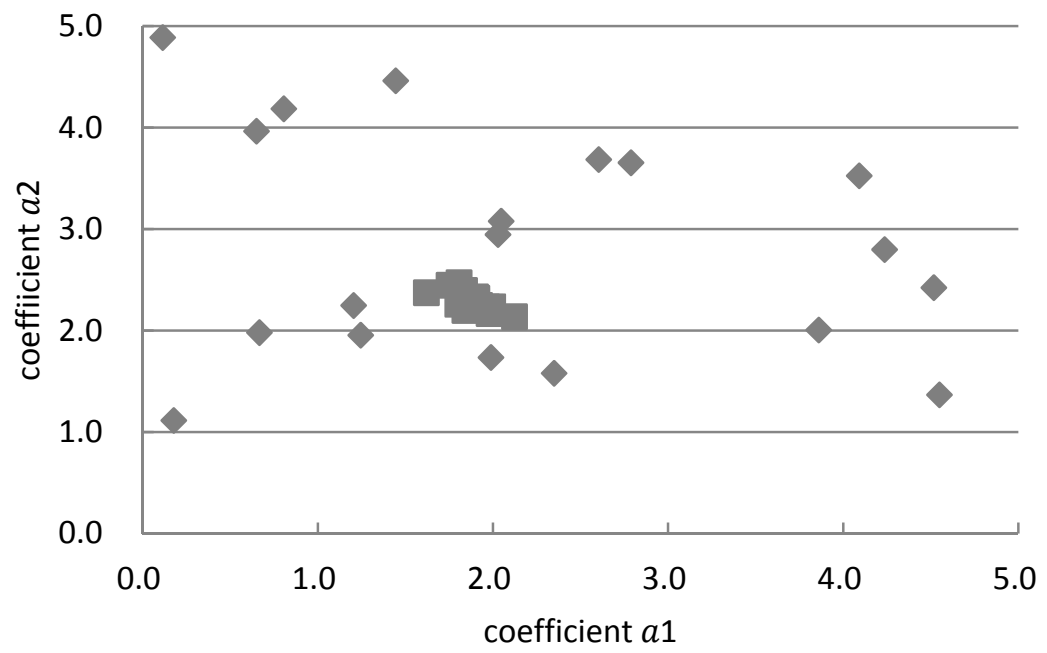

Figure 6: Distribution of employed bees at the initial step and the $100^{\text {th }}$ step in $a_{1} a_{2}$ space. 


\section{Conclusions}

This paper presented one method to carry out inverse analysis in functionally graded materials with the $\mathrm{ABC}$ algorithm. The numerical experiments of inverse analysis were performed by minimizing the objective function consisting of the residual sum of squares of the difference between both the measured quantities and the quantities obtained by numerical analysis. The $\mathrm{ABC}$ algorithm was utilized effectively in the process of minimizing the objective function.

In the first example, the numerical experiment of the inverse analysis of both the graded index and the length of the cantilever FGM beam was conducted and obtained an accurate solution with relatively few iterations. The second example estimated the distribution of thermal conductivity of two-dimensional FGMs based on a distribution of steady state temperatures. The result obtained by the numerical experiment was in good agreement with the correct solution confirming the validity of the proposed method.

Future work will focus on examinations of CPU time in multidimensional problems and additional practical problems. Additionally, how to address noise in measured physical quantities will be explored.

\section{References}

[1] K. Kishimoto et al., Measurement of Elastic Modulus of Functionally Gradient Material Using Inverse Analysis, The Japan Society of Mechanical Engineering(A) Vol. 59, No. 557, pp. 197-202, 1993, in Japanese.

[2] J. Oda and M. Hattori, An Inverse Analysis Technique to Obtain Material Properties Using Strain Data, The Japan Society of Mechanical Engineering(A) Vol. 58, No. 552, pp. 90-95, 1992, in Japanese.

[3] D.E. Goldberg, Genetic algorithms in search, optimization, and machine learning, Addison Wesley, 1989.

[4] D. Dasgupta, Artificial immune systems and their applications, SpringerVerlag, 1999.

[5] K. Price, R. Storn and J. Lampinen, Differential evolution, Springer, 2005.

[6] M. Dorigo and T. Stiutzle, Ant colony optimization, MIT press, 2004.

[7] M. Clerc and J. Kennedy, The Particle Swarm-Explosion, Stability, and Convergence in a Multidimensional Complex Space, IEEE Transactions on Evolutionary Computation, Vol. 6, No. 1, pp. 58-73, 2002.

[8] D. Karaboga, An Idea Based on Honey Bee Swarm for Numerical Optimization, Technical Report-TR06, Erciyes University, Engineering Faculty, Computer Engineering, 2005 (PDF).

[9] D. Karaboga and B. Akay, A comparative study of Artificial Bee Colony algorithm, Applied Mathematics and Computation 214, pp. 108-132, 2009.

[10] P.C. Fourie and A.A. Groenwold, The particle swarm optimization algorithm in size and shape optimization, Struct Multidisc Optim 23, pp. 259-267, 2002. 
[11] G. Venter and J. Sobieszczanski-Sobieski, Particle Swarm optimization, $43^{\text {rd }}$ AIAA/ASME/ASCE/AHS/ASC Structures, structural dynamics, and Materials Conference, April 22-25, pp. 1-9, 2002.

[12] M. Sonmez, Artificial Bee Colony algorithm for optimization of truss structures, Applied Soft Computing 11, pp. 2406-2418, 2011.

[13] S.N. Omkar and J. Senthilnath et al., Artificial Bee Colony (ABC) for multi-objective design optimization of composite structures, Applied Soft Computing 11, pp. 489-499, 2011.

[14] E. Hetmaniok, D. Slota and A. Zielonka, Solution of the Inverse Heat Conduction Problem by Using the ABC Algorithm, Rough Sets and Current trends in Computing Lecture Notes in Computer Science Volume 6086, pp. 659-668, 2010.

[15] Z. Zhong and T. Yu, Analytical solution of a cantilever functionally graded beam, Composite Science and Technology, Vol. 67, pp. 481-488, 2007.

[16] H. Sakurai, Analytical solution of a two-dimensional elastostatic problem of functionally graded materials via the Airy stress function,

[17] WIT Transactions on Engineering Sciences, Vol. 72, pp. 119-130, 2011.

[18] Ochiai, Y., Two-dimensional steady heat conduction in functionally gradient materials by triple-reciprocity boundary element method, Engineering Analysis with Boundary Elements 28, pp. 1445-1453, 2004.

[19] H. Sakurai, Transient and steady-state heat conduction analysis of twodimensional functionally graded materials using particle method, WIT Transactions on Engineering Sciences, Vol. 64, pp. 45-54, 2009.

[20] Koshizuka, S. and Oka, Y., Moving-Particle Semi-implicit Method for Fragmentation of Incompressible Fluid, Nucl. Sci. Eng., 123, pp. 421-434, 1996.

[21] H. Sakurai, Two numerical methods based on discretization of the MPS method for solving the two-dimensional Burgers' equations,

[22] WIT Transactions on Modeling and Simulation, Vol. 55, pp. 79-90, 2013. 